\title{
Vacuum Infusion Process Development for Conformal Ablative Thermal Protection System Materials
}

\author{
Adam T. Sidor ${ }^{1}$ \\ Georgia Institute of Technology, Atlanta, GA, 30332 \\ Robert D. Braun ${ }^{2}$ \\ University of Colorado, Boulder, CO, 80309 \\ Robin A. Beck ${ }^{3}$ \\ NASA Ames Research Center, Moffett Field, CA, 94035 \\ Margaret M. Stackpoole ${ }^{4}$ \\ NASA Ames Research Center, Moffett Field, CA, 94035
}

\begin{abstract}
Conformal ablators are low density composite materials comprised of a flexible carbon felt based fibrous substrate and a high surface area phenolic matrix. These materials are fabricated to near net shape by molding the substrate, placing in a rigid matched mold and infusing with liquid resin through a vacuum assisted process. The open mold process, originally developed for older rigid substrate ablators, such as PICA, wastes a substantial amount of resin. In this work, a vacuum infusion process - a type of liquid composite molding where resin is directly injected into a closed mold under vacuum - is advanced for conformal ablators. The process reduces waste over the state-of-the-art technique. Small, flat samples of Conformal Phenolic Impregnated Carbon Ablator are infused using the new approach and subjected to a range of curing configurations and conditions. Resulting materials are inspected for quality and compared to material produced using the standard process. Lessons learned inform subsequent plans for process scale up.
\end{abstract}

\section{Introduction}

Conformal ablators, first introduced in the early 2000s under the NASA Hypersonics Project $\backslash$ cite $\{$ Gasch2016 $\}$ and developed over the last five years at NASA Ames Research Center with funding from NASA's Science and Technology Mission Directorate (STMD) Game Changing Development Program (GCDP), can alleviate engineering and processing challenges typical of rigid ablative thermal protection system (TPS) materials. Unlike the rigid substrates used in Phenolic Impregnated Carbon Ablator (PICA) and related materials, conformals use flexible fibrous substrates, such as felts or battings) which, after processing, yield rigid TPS materials with higher strain to failure than their rigid substrate counterparts. The flexible substrates are conformed in a mold to produce near net shape parts with consistent fiber orientation, which results in uniform thermal properties through the thickness. By contrast, a curved part machined from a rigid block will have variable fiber orientation, and thus non-optimal through thickness properties (e.g., thermal conductivity). Near net shape manufacturing and higher strain to failure allow conformal ablators to be manufactured as larger TPS tiles, thereby reducing part count, and allowing for installation directly on most aeroshells

1 Graduate Research Assistant, Guggenheim School of Aerospace Engineering, AIAA Student Member.

2 Dean, College of Engineering and Applied Science, AIAA Fellow.

3 Conformal Ablative TPS project lead,_-Entry Systems and Vehicle Development Branch, AIAA Associate

Fellow.

4 Senior Research Scientist, Thermal Protection Materials Branch, AIAA status. 
without strain isolation layers. In addition, the higher strain to failure behavior of the conformal TPS may allow the designers to eliminate gaps and gap fillers between tiles. Reduced part count and simplified integration drive reductions in labor, cost and complexity $[2,3,4,5,6,7,1]$ - advancements which are enabling for planetary and human missions $[8,9,10]$.

State of the art manufacturing for conformal ablative materials uses an open liquid impregnation process adapted from PICA processing in which the fibrous substrate, constrained in the mold, is infused using a vacuum assisted infusion approach $[6,1]$. Mold openings are required for proper infiltration of the substrate. Some resin necessarily remains outside the substrate, but is cured along with the part and then trimmed away from the mold and discarded post process. A closed infusion process, where resin is injected directly into the substrate, can eliminate much of this waste, reducing cost and labor. Liquid composite molding (LCM) processes have been in use in composites manufacturing for decades and do just that. These processes drive resin into a fibrous substrate within a matched mold through an applied pressure differential. Resin delivery is accomplished through a variety of mechanisms depending on the specific process. A vacuum infusion process (VIP) applies vacuum to a mold cavity while atmospheric pressure forces resin into the enclosed substrate. In this work, several small samples of Conformal Phenolic Impregnated Carbon Ablator (C-PICA) are fabricated using VIP. Carbon felt is infused with a dilute phenolic resin in a mold and then subjected to a range of curing configurations and conditions to determine the best approach. Resulting TPS materials are visually inspected for resin uniformity and voids. Then, relevant physical properties are compared to C-PICA produced using the standard process. Finally, lessons learned from the present testing are summarized and applied to plans for scaling up VIP to a larger, curved TPS tile.

\section{Background and Motivation}

\subsection{Composition of Conformal Ablators}

Conformal ablative TPS materials are composites consisting of a flexible fibrous substrate and polymer matrix, similar to other fiber-reinforced plastics. Unlike structural composites, conformals possess relatively low density and fiber fraction (around $10 \%$ [1]) by design ${ }^{1}$. Resins are substantially diluted to control resin loading and allow for infusion at lower pressures, producing a lightweight, porous material. Several conformal TPS variants have been developed. CPICA and C-SIRCA are conformal analogs of PICA and SIRCA (Silicone Impregnated Reusable Ceramic Ablator), respectively. C-PICA combines a high porosity rayon-based carbon felt insulator with phenolic resin while C-SIRCA consists of a silica based felt substrate and silicone resin $[4,10]$. While the density of C-PICA is similar to that of PICA, C-PICA is more thermally efficient which reduces required TPS mass [7]. Recent work has focused on identifying alternative carbon felt substrates manufacturable in larger thicknesses and higher densities[1].

\subsection{State-of-the-Art Processing}
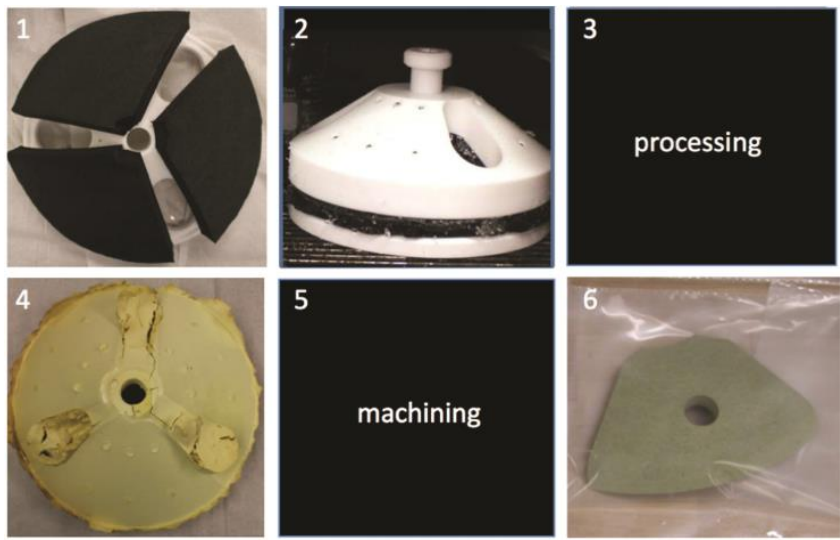

Figure 1: State-of-the-art fabrication process for conformal ablators (Credit: Ref. Milos2015).

\footnotetext{
${ }^{1}$ Fiber consolidation/compaction would compromise thermal performance by increasing thermal conductivity
} 
Conformal ablators are manufactured using a modified liquid impregnation procedure originally developed for PICA, SIRCA, and related rigid substrate ablators $[1,6,11]$. Several variations on the process are outlined in a patent for those materials[12]. Generally, a fiber substrate is infused using a vacuum assisted infusion process. The material is cured and then dried to remove residual solvent. Temperature and pressure profiles are resin-dependent, and multiple cycles may be required to complete curing and solvent removal. For some materials, impregnation and curing/drying is carried out multiple times to increase resin loading. Conformal ablators conform the substrate in a mold prior to impregnation yielding a near net shape part..

The complete process is depicted in Figure 1 [6]. First, (1) the substrate is cut and formed to the desired geometry, then (2) draped within a matched mold. Next, (3) the material is processed using liquid impregnation producing (4) a rigid, near net-shape part. Finally, (5) excess material is removed by machining yielding (6) the final TPS tile. Note that processing and machining are not shown due to the proprietary nature of these techniques. However, it is sufficient to understand that the liquid impregnation procedure is an open process as described above - the mold is not closed, or sealed, but rather is partially open to its surroundings (see Step (2) of Figure 1). Molds require a large degree of open space to allow resin to enter and ensure complete infusion. Excess resin outside the substrate must be cured along with the part only to be later removed and discarded. This de-molding and clean up adds time and effort to processing in addition to wasting resin. [Mairead: Double check details on current process.]

\subsection{Liquid Composite Molding}

In liquid composite molding (LCM), resin is injected into a fibrous substrate enclosed in a cavity within a matched mold. Resin enters through one or more inlets driven by a positive pressure differential. Air and other volatiles are removed, or forced out, at one or more vents. The resin is then cured to produce a rigid composite material. LCM, in some form, has existed at least since the 1940s. The Marco method, designed as an improvement over hand layup for boat hulls, is generally cited as the first use of LCM [13,14, 15]. In the Marco method, vacuum draws resin into a fibrous substrate sealed between a semi-flexible bag and a rigid mold. LCM soon expanded into the aerospace industry [16] in the 1950s and, subsequently, the rail, automobile and marine industries[13, 15].

Today, LCM encompasses a variety of processes including gravity and vacuum infusion, resin transfer molding (RTM), vacuum assisted resin transfer molding (VaRTM), structural reaction injection molding (SRIM), and many others. Mold tooling may be rigid or flexible. Resin curing may occur during mold filling (to reduce cycle time) or after filling is complete. Some processes use a pump to drive resin (RTM), others use vacuum, and some use both (VaRTM). While the details of these processes differ, all share basic similarities: a resin delivery system, a fiber handling system, a matched mold and some approach to controlling resin flow and air removal[13]. Another author identifies the extent, and importance, of resin flow as the primary characteristic differentiating LCM from other composite manufacturing processes[17]. Ultimately, all LCM processes accomplish the same end - driving liquid resin into a dry, fiber preform through an applied pressure differential.

\subsection{Motivation}

Conformal TPS manufacturing appears to be a natural extension of LCM. The construction of conformal ablators is remarkably similar to other fiber-reinforced plastics currently manufactured using LCM, yet state-of-the-art fabrication relies on the liquid impregnation approach of older rigid substrate resin impregnated ablators. A substantial amount of resin is wasted in this open process. LCM can advance conformal ablator manufacturing twofold: (1) by directly injecting resin into a closed mold thus eliminating waste and (2) by allowing numerical simulation to be introduced into TPS manufacturing. Resin flow can be numerically simulated to facilitate mold and process design. Such an approach can identify potential issues, e.g., areas of poor infusion, in advance and lead to improved designs.

In this work, a vacuum infusion process (VIP) is advanced for manufacturing conformal ablative TPS. VIP, a subset of LCM, uses vacuum to evacuate air and draw resin into the fiber substrate. The vacuum-driven process is suited to the low viscosity resins and high porosity, high permeability substrates typical of conformal ablators. Evacuation of the cavity limits the risk of air entrapment during mold filling, which can form voids and be problematic when using certain resin systems. In other LCM processes, entrapped air is typically compressed and driven out of the part through compaction pressure, which is undesirable for these low density TPS materials. Low working pressure leads to a relatively simple process - one that is straightforward to set up and requires only a basic set of flow control hardware - and does not require high strength, high stiffness molds. 


\section{Vacuum Infusion Process}

The vacuum infusion process encompasses three steps: (1) infusion, (2) curing, and (3) drying (Figure 2). Two rigid mold sides constrain the part during infusion and curing. The cavity is sized such that little to no compaction is applied to the substrate. Limiting substrate compaction is necessary to ensure uniform part density. Resin infusion is achieved by drawing vacuum on the mold cavity and allowing atmospheric pressure to drive resin into the substrate. A perimeter gasket seals the two mold halves. Once the cavity is evacuated, resin is introduced into the cavity via an inlet. Resin flows through the substrate, driven by the pressure differential, until the cavity is completely filled. Vacuum is continually pulled on the cavity until completion. The entire assembly, with saturated substrate inside, is then transferred to an oven for curing. One or more ports allow volatiles to escape the cavity during this step. Once cured, the now rigid part is removed from the mold, returned to an oven, and dried.

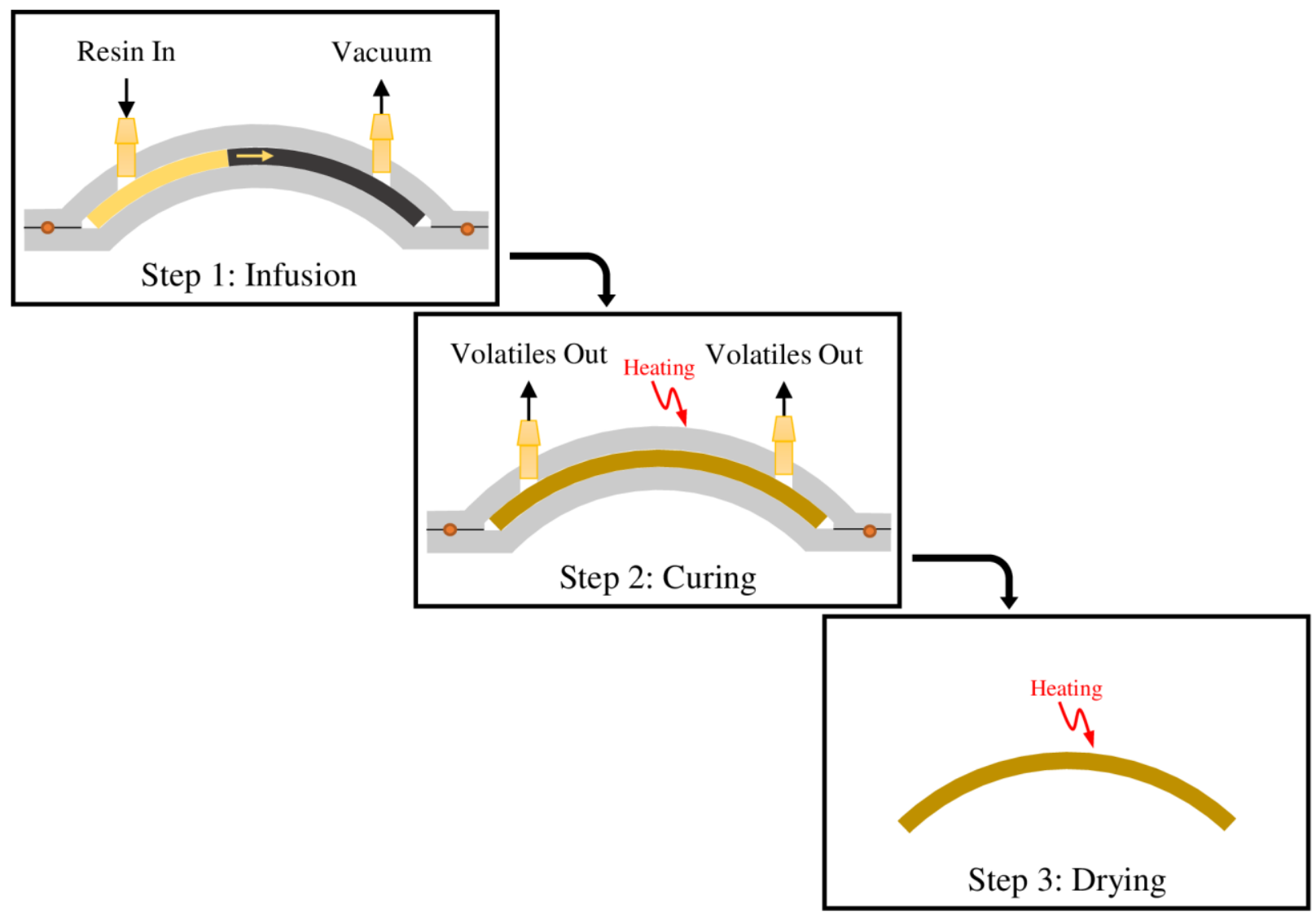

Figure 2: Overview of vacuum infusion process.

\section{Experimental Methodology}

\subsection{Material Formulation}

The present testing is carried out on a C-PICA formulation consisting of carbon felt (Morgan Advanced Materials VDG Carbon Felt) and a proprietary phenolic resin solution. The felt has nominal bulk density of $0.09 \mathrm{~g} / \mathrm{cc}$. Resin loading is approximately $0.2 \mathrm{~g} / \mathrm{cc}$ resulting in a TPS density of around $0.29 \mathrm{~g} / \mathrm{cc}$. In practice, the substrate density varies between $0.08 \mathrm{~g} / \mathrm{cc}$ and $0.10 \mathrm{~g} / \mathrm{cc}$ producing corresponding variability in TPS density. 


\subsection{Processing}

A two-part aluminum mold encloses the material during processing (Figure 3). Note that the clear top, shown here for visibility, was replaced with an aluminum lid for processing. The substrate resides in a 4" x 4" square cavity, 0.85 " deep, machined into the bottom part. The cavity is PTFE-coated to ease demolding. A compressible O-ring gasket seals the perimeter of the cavity between the top and bottom parts. Two ports in the top allow flow into and out of the cavity. A series of pipe couplers and flow control hardware, the port sub-assembly, adapt these openings to external hardware. Port configuration, described below and depicted in Figure 4, is specific to the processing step.
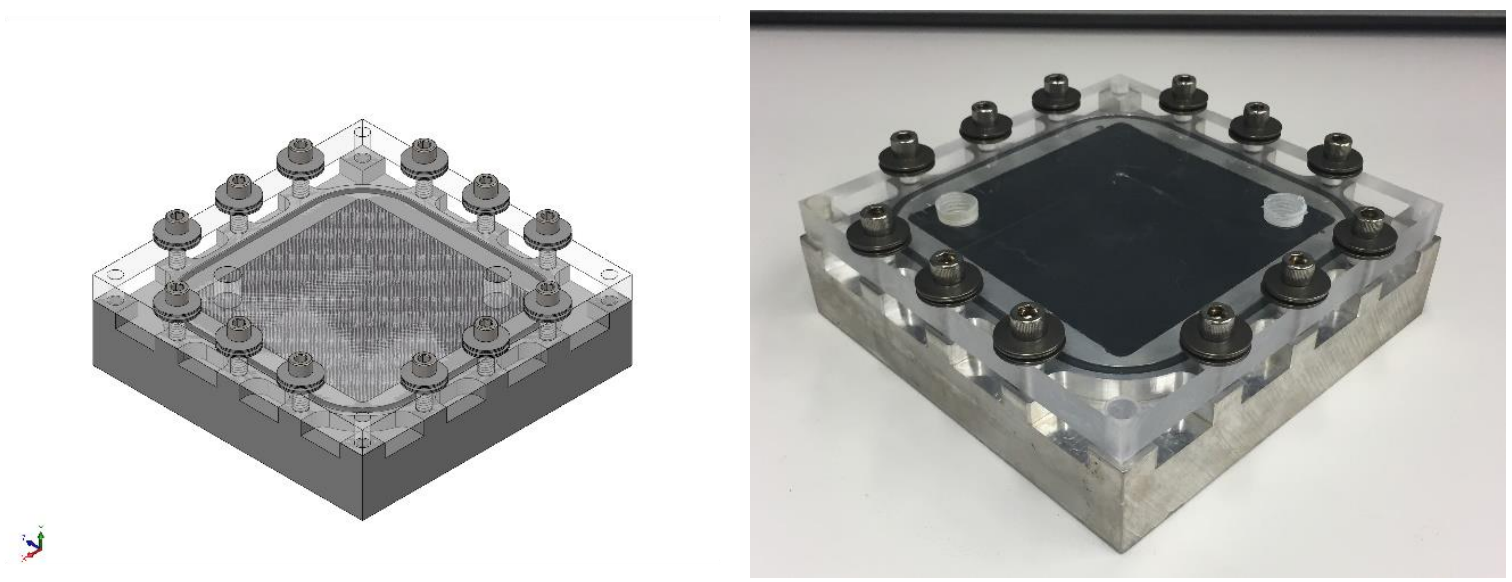

Figure 3: Test mold as designed (left) and as fabricated (right), without port assemblies.

\subsubsection{Infusion}

During infusion, the port sub-assembly consists of a shut off valve and a barbed tube fitting (Figure 4, left). The two ports are connected via tubing to a resin reservoir and a vacuum pump, respectively. A resin trap between mold and pump collects overflow (Figure 5). With the vacuum pump on and both ports open, atmospheric pressure drives resin into the tubing resting at the bottom of the reservoir. The resin enters the mold (and substrate) through the inlet and then exits through the outlet and into the resin trap. Once resin reaches the trap, the shut off valves are closed to stop flow.
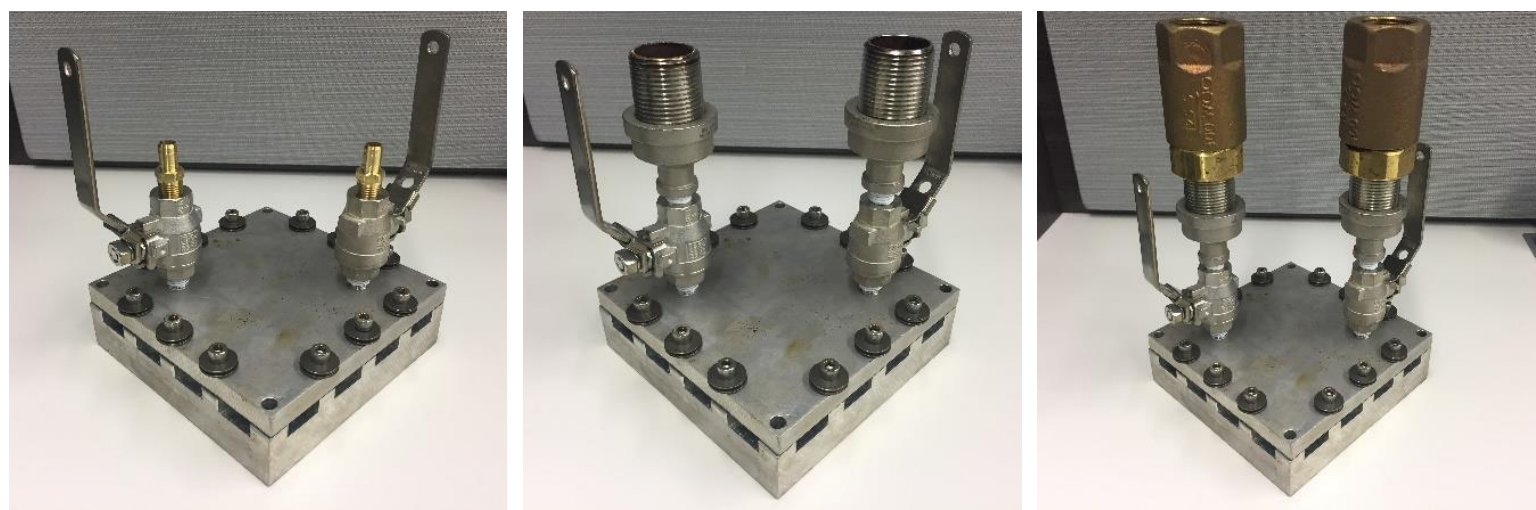

Figure 4: Port configurations during processing (left to right): infusion, open curing, closed curing. 


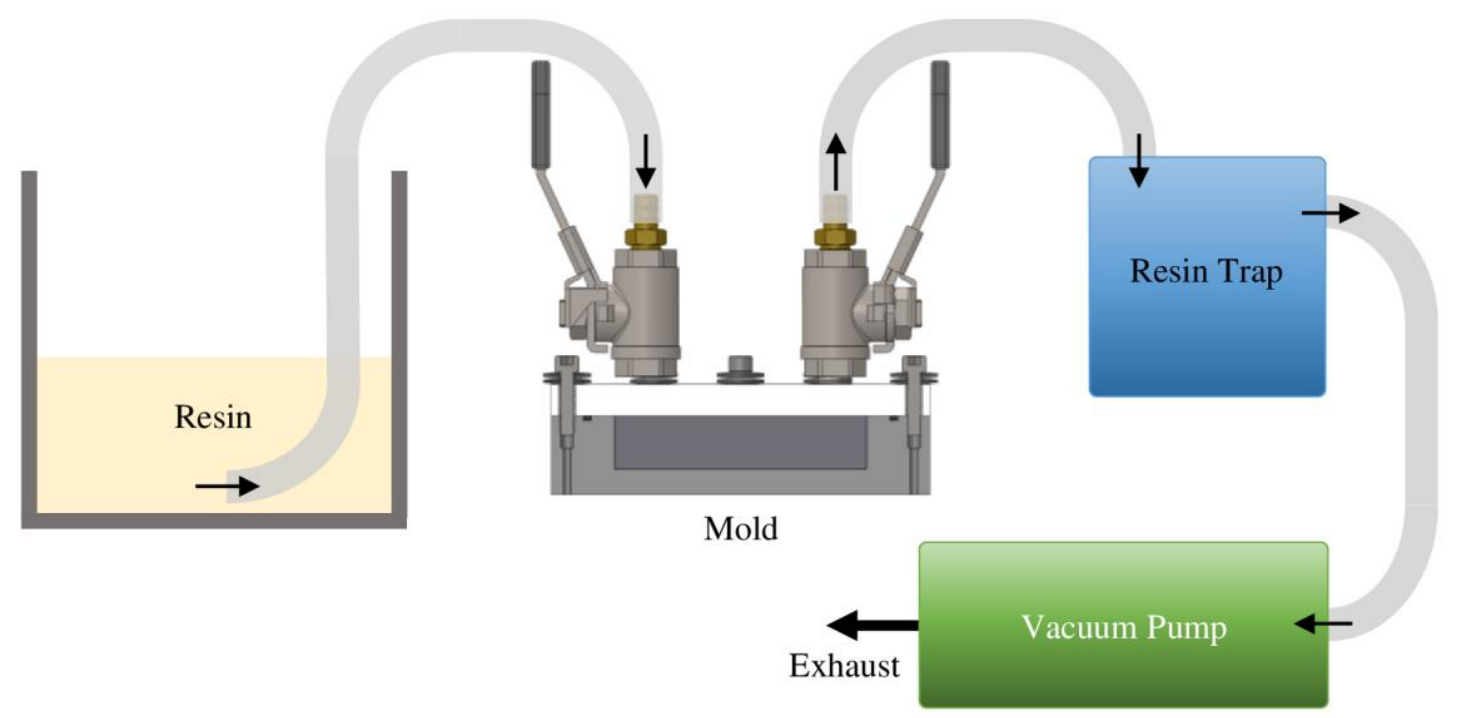

Figure 5: Diagram of the infusion set up.

\subsubsection{Curing}

The port sub-assembly is reconfigured after infusion and prior to curing. The barbed fitting is removed and additional pipe couplings installed. These couplings adapt the opening from a small diameter to a large diameter. The additional volume accommodates thermal expansion of the resin (and thus a rise in liquid level) during curing, preventing overspill. In certain test cases, the port is open to the ambient oven atmosphere during curing (Figure 4, center). In other tests, a one-way check valve is installed at the top of the stack to control the flow of volatiles and prevent air ingress (Figure 4, right). These two configurations are designated open, in the former, or closed, in the latter.

\subsection{Test Matrix}

One C-PICA coupon was fabricated using the standard open infusion process. Five C-PICA coupons were fabricated using the formulation and VIP setup above. Test conditions are summarized in Table 1. In addition to port configuration, the ambient atmosphere during curing (pressure and composition) was also varied. In two of the three closed configuration tests, curing was performed in air, rather than an inert atmosphere, with the assumption that the check valves would prevent backflow into the cavity and subsequent resin oxidation. One open curing test was performed at reduced pressure - under the hypothesis that lower ambient pressure might yield better volatile removal and lower void content. Note that for closed configurations, the ports were evacuated prior to curing. In one case (Sample 6), the port was backfilled with inert gas. In the other two cases, the ports were under vacuum initially, but it is assumed that evaporating solvent rapidly filled these small volumes.

Table 1: Matrix of test conditions.

\begin{tabular}{c|c|cccc}
\hline \multirow{2}{*}{$\begin{array}{c}\text { Sample } \\
\text { Number }\end{array}$} & \multirow{2}{*}{ Process } & $\begin{array}{c}\text { Port } \\
\text { Oven }\end{array}$ & $\begin{array}{c}\text { Oven } \\
\text { Configuration }\end{array}$ & Pressure & Atmosphere \\
\hline 1 & Standard & - & $1 \mathrm{~atm}$ & Inert & - \\
2 & VIP & Open & $1 \mathrm{~atm}$ & Inert & - \\
3 & VIP & Closed & $1 \mathrm{~atm}$ & Air & No \\
4 & VIP & Closed & $1 \mathrm{~atm}$ & Inert & No \\
5 & VIP & Open & $0.66 \mathrm{~atm}$ & Inert & - \\
6 & VIP & Closed & $1 \mathrm{~atm}$ & Air & Yes \\
\hline
\end{tabular}




\subsection{Material Characterization}

\subsubsection{Testing and Measurement Procedure}

\section{Visual Inspection}

TPS materials were visually inspected to assess the uniformity of resin infusion, presence (or absence) of voids, and substrate delamination. Cured phenolic (a characteristic yellow) should be distributed uniformly through the material thickness and fill all the void area. Voids, areas with little or no resin, and delamination are both undesirable due to their compromising effect on TPS performance. Delamination, separation of felt layers, can occur due to curing stresses in the resin as it shrinks during process - the substrate does not have sufficient integrity to resist the shrinkage. Note that external surfaces are not necessarily indicative of through the thickness infusion. Frequently, this nonuniformity is isolated to the surface of the part and would be removed in post-processing machining. However, nonuniformity and/or voidage extending into the bulk of the material is detrimental.

Two types of voids are typically encountered during processing. The first are larger and tend to appear in depth (Figure Error! Reference source not found., left); the latter are smaller but distributed near the top surface (Figure Error! Reference source not found., right). Note that pictured materials are cores drilled through the thickness showing in depth material. The larger voids likely result from air entrapment during infusion which leave dry, unsaturated pockets in depth. The smaller voids along the top surface stem from solvent evaporation during curing. Premature solvent evaporation causes the resin to "collapse", that is, to no longer be suspended in solution leaving dry areas near the top surface.
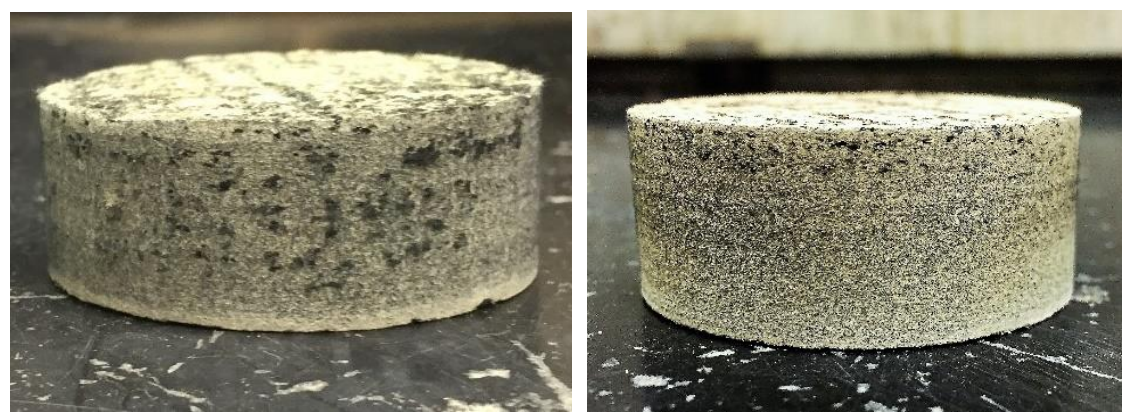

Figure 6: Void formation during processing: larger voids due to entrapped air in depth (left) and smaller voids near top surface due to solvent evaporation (right).

\section{Substrate Density}

Substrate density, in the composite material, is an important driver of bulk TPS properties. Compression and shrinkage during processing mean that this density is actually higher than that of the raw felt. The final (average) substrate density is estimated by dividing the initial (dry) substrate mass by the final TPS volume.

\section{Virgin Density}

Virgin TPS density is computed from 2-inch diameter coupons drilled through the thickness of each sample. Each coupon was sanded to remove roughness and variability near the top and bottom surfaces. Coupon thickness was subsequently reduced by 2 to 3 millimeters in total.

\section{Char Yield}

Char yields were obtained by heating each 2-inch diameter coupon to $1200 \mathrm{deg} \mathrm{C}$ (3C/min with a $30 \mathrm{~min}$ hold) in an inert furnace. Yields for both the composite material and the resin itself are computed as well as pre- and post-charring dimensions. The char yield of the composite is related to the amount of phenolic present in the material. Higher resin 
loading results in greater relative mass loss during charring and a lower char yield. However, there also exists a dependence on the structure of the phenolic [18].

\subsubsection{Analysis}

Relevant material properties (virgin density, char yield and resin mass fraction) are computed and compared to expected values from a geometric material model. The analysis accounts for substrate variability, specifically pore volume, on TPS properties.

\section{Substrate Model}

The substrate is modeled as a network of smooth, nonporous cylindrical fibers of uniform density. Such an approach, the filamentary analog method, has been applied elsewhere to estimate porosity, permeability, and specific surface area of low density carbon felts similar to the present work[19]. Employing the filamentary analog model simplifies preform properties to functions of density. Porosity, $\varphi$, is estimated as a ratio of bulk (or apparent) substrate density, $\varrho_{\text {sub }}$, to fiber density, $\varrho_{\text {fiber }}$.

$$
\varphi=1-\frac{\varrho_{\text {sub }}}{\varrho_{\text {fiber }}}
$$

where $\varrho_{\text {sub }}$ is a globally-averaged quantity such that the substrate mass is

$$
m_{\text {sub }}=\varrho_{\text {sub }} V_{\text {bulk }}
$$

Bulk volume, $V_{\text {bulk }}$, measured from external dimensions, is the total volume of fibers and pores. Fiber density, $\varrho_{\text {fiber' }}$ of Morgan VDG felt is $1.395 \pm 0.055 \mathrm{~g} / \mathrm{cc}$ (95\% confidence interval, see Appendix). Note that this value is in line with other data on rayon-based carbon fibers. Ref. Pierson1993 suggests a range from $1.40 \mathrm{~g} / \mathrm{cc}$ to $1.80 \mathrm{~g} / \mathrm{cc}$ though the upper end encompasses high modulus fibers that are no longer manufactured. Ref. Morgan2005 lists commercially available fibers from $1.35 \mathrm{~g} / \mathrm{cc}$ to $1.44 \mathrm{~g} / \mathrm{cc}$. Pore volume, the amount of empty space within the substrate, follows from porosity,

$$
V_{\text {pore }}=\varphi V_{\text {bulk }}
$$

\section{TPS Model}

Expected TPS properties are computed as functions of fiber density, pore volume, and resin density. The former two, fiber density and pore volume, are described above. Pore volume has an obvious impact on the TPS material: more pore volume allows more resin to infiltrate the substrate. Resin density, $\varrho_{\text {resin }}$, is a globally-averaged quantity such that

$$
m_{\text {resin }}=Q_{\text {resin }} V_{\text {pore }}
$$

where $m_{\text {resin }}$ is the total resin mass in the TPS after processing. Note that $m_{\text {resin }}$ is a function of the pore, not bulk, volume. Substituting Equations (1) and (3) into Equation (4) yields

$$
m_{\text {resin }}=\varrho_{\text {resin }}\left(1-\frac{\varrho_{\text {sub }}}{\varrho_{\text {fiber }}}\right) V_{\text {bulk }}
$$

With known resin char yield, $Y_{\text {resin }}^{c}$, char mass can also be computed,

$$
m_{\mathrm{char}}=m_{\mathrm{sub}}+Y_{\mathrm{resin}}^{c} m_{\text {resin }}
$$

Table 2: Expressions for TPS property estimation. 


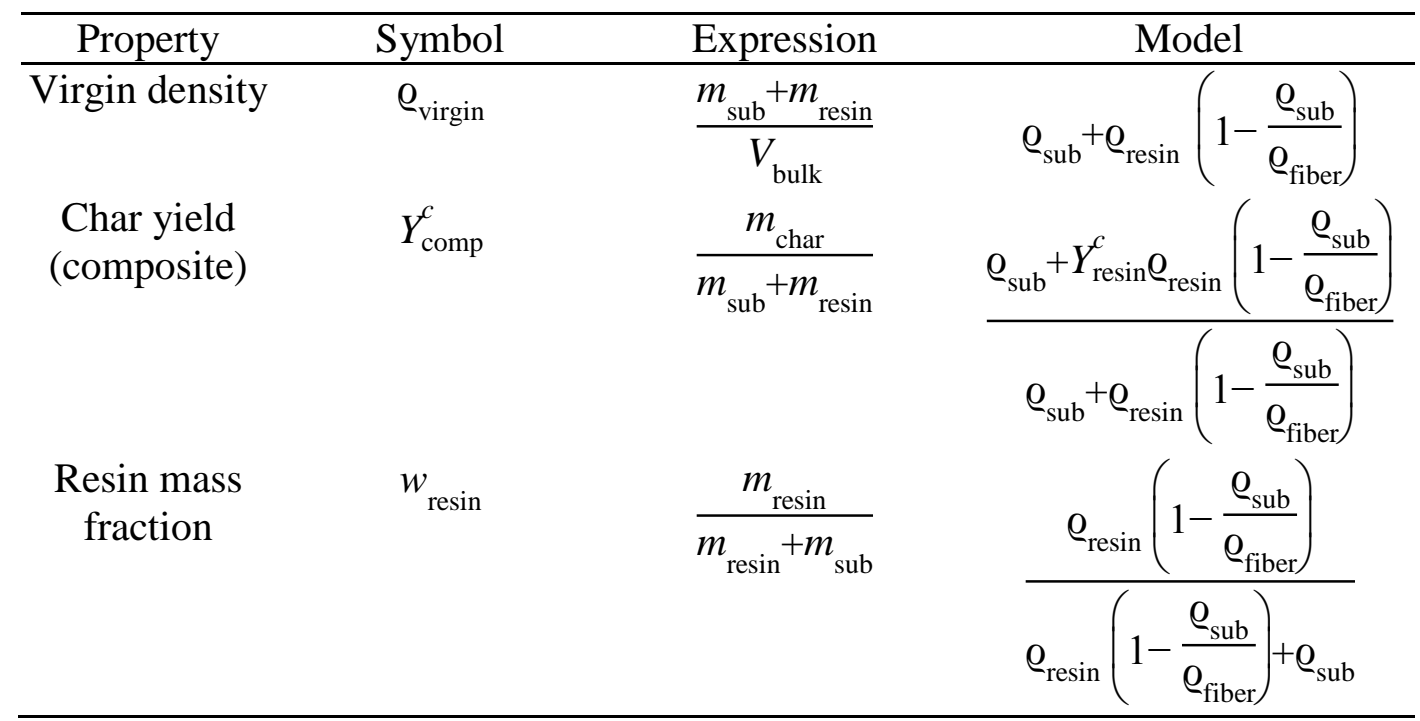

The relevant TPS properties are computed according to the expressions in Table 2. Equations (1)-(6) reduce these expressions to functions of density. Note that experimental values of $\varrho_{\text {resin }}$ and $Y_{\text {resin }}^{c}$ derived from Sample 1 are used here. Thus, VIP-fabricated coupons are directly compared against material produced via the standard process.

\section{Results}

\subsection{Sample Processing}

Substrate materials were cut from a single 41" by 12" piece of Morgan VDG carbon felt. The samples were infused at room temperature with phenolic resin. A single resin mixture was used for all samples to avoid variation in resin composition. Once the felt was enclosed within the mold, the cavity was evacuated. Samples 3-6 were held under vacuum for several minutes prior to infusion to allow sufficient time for air removal and outgassing. Sample 2 was not held under vacuum for a significant amount of time (less than 1 minute) which may have resulted in only partial evacuation. Thus, the procedure was adjusted for the subsequent samples.

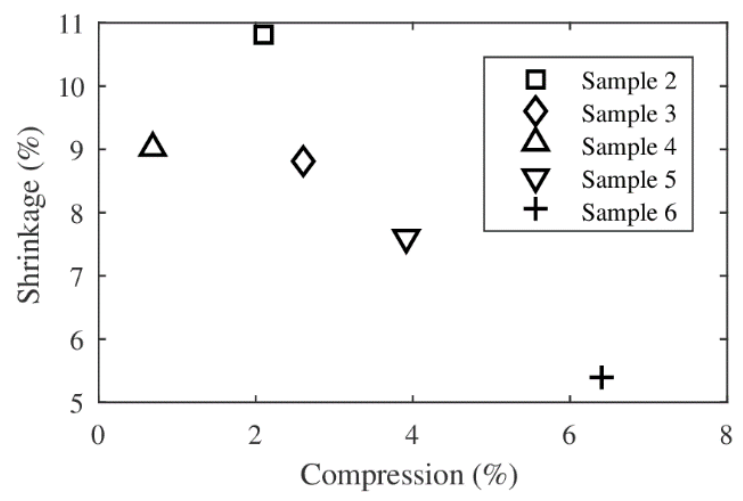

Figure 7: Shrinkage in part thickness as a function of compression (quantities are averaged over the sample).

Once evacuated, the resin valve was opened (with the vacuum valve still open) to allow resin to enter the substrate. It was observed that resin advanced rather quickly from inlet to outlet, especially if the resin valve was opened quickly, with filling times on the order of only a few seconds. It was also found that in processing, bubbles were generated 
during infusion (observed once resin reached the outlet). These bubbles could be the result of solvent flashing and/or boiling - the former as the resin encounters a rapid pressure drop upon first entering the inlet and the latter along the flow front while inside the mold. This bubble formation did not appear to produce permanent voids in the final material.

Once filled, both valves were closed to stop resin flow. The ports were then reconfigured according to Table 1 and transferred to an oven for curing. While some parts (Sample 3 and 6) were cured in air with the mold presumably sealed, it was found after curing that air had entered through the port causing oxidation. Fortunately, oxidation occurred at the ports only and did not extend to the TPS material itself. After curing, the parts were removed from the mold and returned to the oven for drying.

Sample masses and dimensions recorded before infusion (the initial, uncompressed felt) and after processing are summarized in Table 3. The mold cavity is slightly undersized, 3.9965" x 3.9955" x 0.8533" deep) thus the felt substrates were compressed by around $0.5 \%$ in width and length and a few percent in thickness (Samples 2-6). Sample 1 , which was processed outside of a mold using the standard process, was compressed approximately $0.6 \%$ in thickness - a weight was placed on top of the felt to keep it submerged during infusion. Note the change in dimensions that occurs during processing, from the initial felt to the final TPS. In general, the part shrinks during curing and, then, expands slightly during drying. For the VIP-fabricated samples, there is a net decrease in all dimensions from the initial felt. Sample 1 increased slightly in width and length (about $1 \%$ ) while decreasing in thickness by about $4 \%$. Changes in thickness are generally larger (5\%-10\%) than those in width and length (typically <2\%, though Samples 5 and 6 shrunk substantially more in length and little in width) due to directionality in the felt fiber structure. Thickness shrinkage appears to be related, at least in part, to the amount of compression applied to the felt (Figure 7). As more compression is applied, the amount of shrinkage tends to decrease.

Table 3: Masses and dimensions of the initial, uncompressed felt and final TPS sample (Note: thickness, $t$, is an average over the sample).

\begin{tabular}{c|cccc|cccc} 
& \multicolumn{4}{|c|}{ Felt Substrate (uncompressed) } & \multicolumn{4}{c}{ TPS Sample } \\
\hline $\begin{array}{c}\text { Sample } \\
\text { Number }\end{array}$ & $\mathrm{m}(\mathrm{g})$ & $\mathrm{W}(\mathrm{in})$ & $\mathrm{L}(\mathrm{in})$ & $\mathrm{t}(\mathrm{in})$ & $\mathrm{m}(\mathrm{g})$ & $\mathrm{W}($ in) & $\mathrm{L}(\mathrm{in})$ & $\mathrm{t}(\mathrm{in})$ \\
\hline 1 & & & & & & & & \\
2 & 18.6462 & 4.0050 & 4.0095 & 0.8944 & 66.8572 & 4.0440 & 4.0400 & 0.8561 \\
3 & 18.6642 & 4.0110 & 4.0015 & 0.8705 & 62.5986 & 3.9840 & 3.9350 & 0.7614 \\
4 & 18.6451 & 4.0245 & 4.0165 & 0.8766 & 62.3191 & 3.9945 & 3.9420 & 0.7781 \\
5 & 19.1030 & 4.0130 & 4.0065 & 0.8543 & 62.9879 & 4.0000 & 3.9280 & 0.7768 \\
6 & 19.3583 & 4.0085 & 4.0035 & 0.9115 & 61.7157 & 3.9960 & 3.8130 & 0.8074 \\
\hline
\end{tabular}

\subsection{Material Characterization}

\subsubsection{Visual Inspection}

A visual inspection of external part surfaces reveals some variation (Figure 8). In the VIP samples, Sample 2-6, the inlet is located at bottom left and outlet at top right. Sample 1, produced using the standard process, is well-infused on all surfaces - an expected result considering the entire substrate is immersed in resin including the edges. Of the VIP materials, Samples 3-6 exhibit surface voidage while Sample 2 does not. Sample 2 used the thinnest felt piece of those processed, which may have allowed the top surface to remain submerged below the resin solution throughout processing. Sample 2 however does possess isolated pockets of oxidation on the surface (dark brown) perhaps from poor evacuation of the cavity prior to infusion. Surface voidage of Samples 3 and 6 appear to be the small voids indicative of solvent evaporation. Voidage on Samples 4 and 5 is larger and appears to be related to felt variation rather than processing effects. 


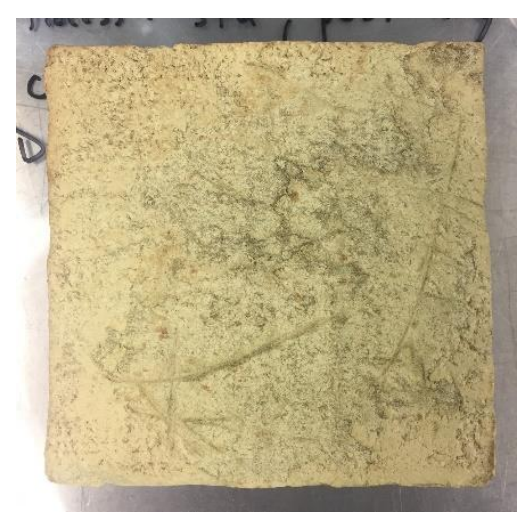

Sample 1

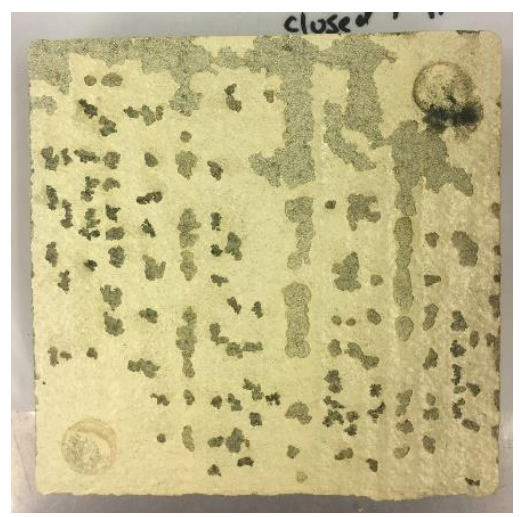

Sample 4

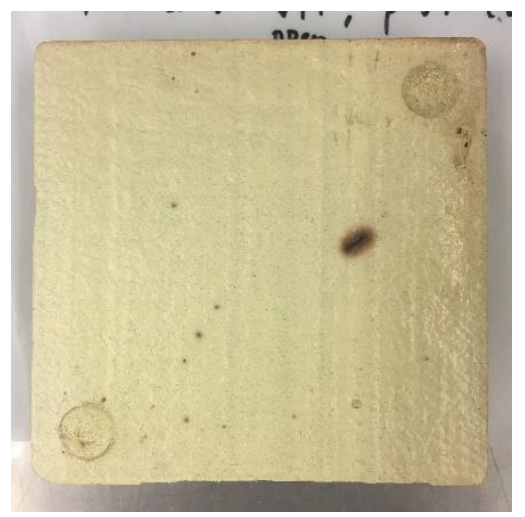

Sample 2

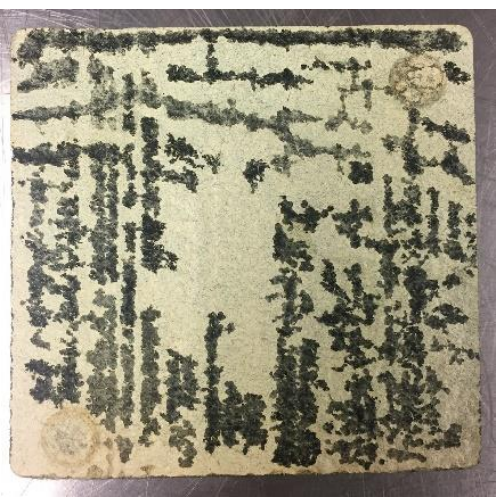

Sample 5

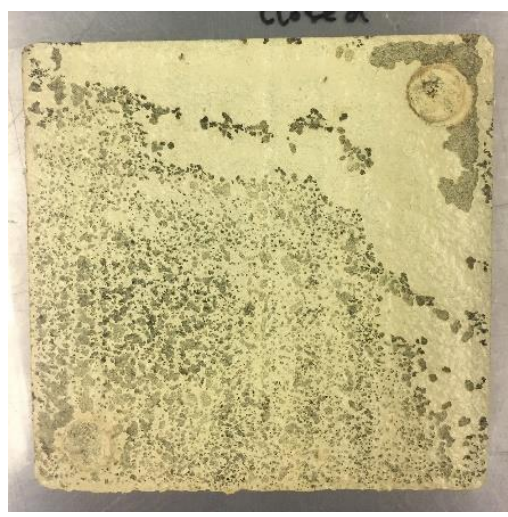

Sample 3

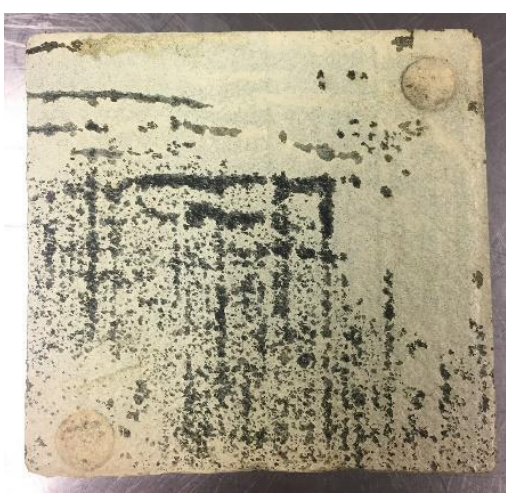

Sample 6

Figure 8: Full TPS samples, top surface only

Cores drilled through the thickness show in depth material (Figure 9). Cores are oriented with the top surface up. Sample 1 is again uniformly infused with no voids (horizontal bands are tool marks from drilling, not characteristic of the material itself). Samples 2, 3 and 4 compare favorably, possessing similarly uniform infusion in depth. Sample 3 exhibits some surface voidage near the top of the coupon, again likely from solvent evaporation, but this voidage does not extend significantly in depth. Sample 4 also exhibits some delamination near the bottom, but this area is small. Sample 5 has large voids characteristic of air entrapment as well as delamination. Sample 6 possesses voidage from evaporation near the top surface that also extends into the thickness.

Note that the cores are pictured prior to sanding, so surface nonuniformity visible here was ultimately removed prior to obtaining subsequent results. For example, voidage at the top of Sample 3 was removed, but farther in depth voidage in Samples 5 and 6 was not.

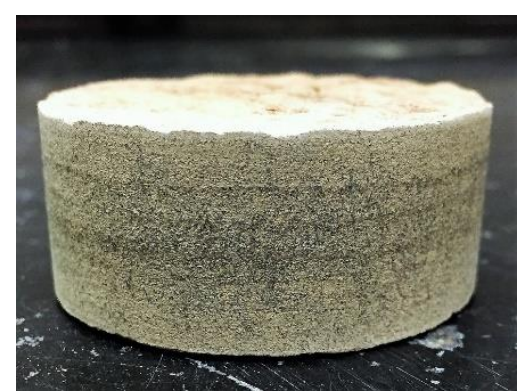

Sample 1

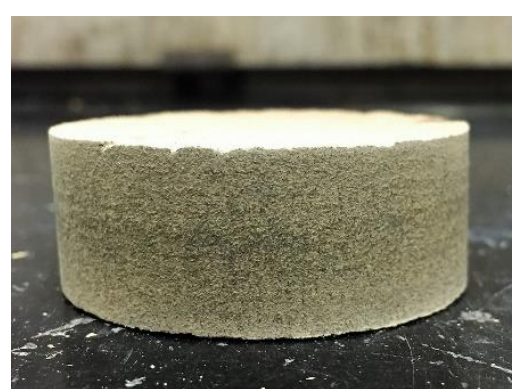

Sample 2

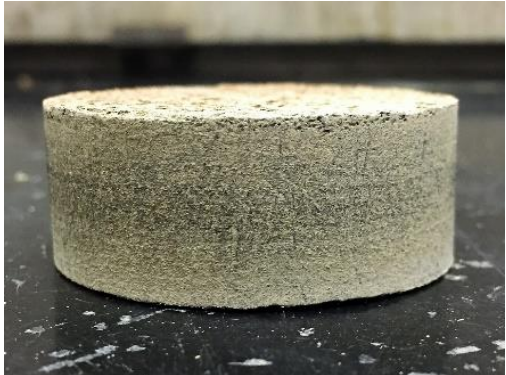

Sample 3 


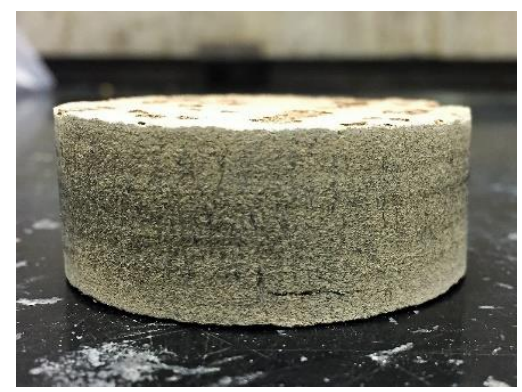

Sample 4

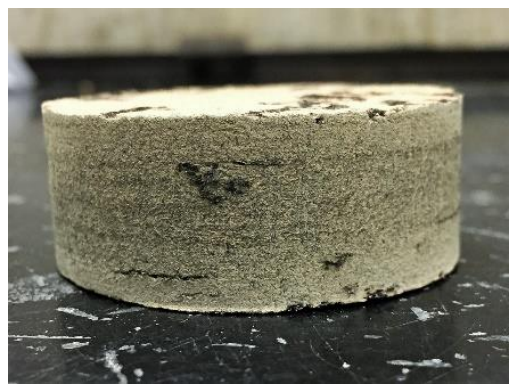

Sample 5

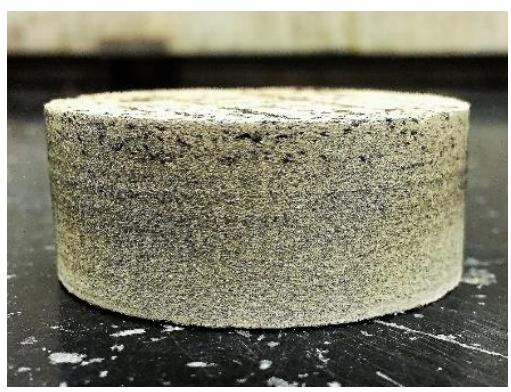

Sample 6

Figure 9: Cored TPS samples, in depth material.

Table 4: Mass and dimensions of cylindrical TPS coupons prior to charring.

\begin{tabular}{c|cc|ccc|cc} 
& \multicolumn{2}{|c|}{ Dimensions } & \multicolumn{3}{|c|}{ Masses } & \multicolumn{2}{c}{ Densities } \\
\hline Sample & $D(\mathrm{~cm})$ & $t(\mathrm{~cm})$ & $m_{\text {resin }}(\mathrm{g})$ & $m_{\text {fiber }}(\mathrm{g})$ & $m_{\text {total }}$ & $\begin{array}{c}\varrho_{\text {substr }} \\
(\mathrm{g} / \mathrm{cc})\end{array}$ & $\begin{array}{c}\varrho_{\text {virgin }} \\
(\mathrm{g} / \mathrm{cc})\end{array}$ \\
\hline 1 & 5.032 & 1.902 & 8.31 & 3.08 & 11.3876 & 0.081 & 0.301 \\
2 & 5.028 & 1.718 & 7.55 & 2.78 & 10.8058 & 0.095 & 0.317 \\
3 & 5.031 & 1.695 & 7.38 & 2.74 & 10.4746 & 0.092 & 0.311 \\
4 & 5.031 & 1.596 & 6.81 & 2.58 & 9.7568 & 0.093 & 0.308 \\
5 & 5.035 & 1.776 & 7.72 & 2.88 & 11.0900 & 0.095 & 0.314 \\
6 & 5.034 & 1.799 & 7.29 & 2.91 & 10.7281 & 0.096 & 0.300 \\
\hline
\end{tabular}

\subsubsection{Substrate and Virgin Density}

Substrate density and virgin TPS density for the cores in Figure 9 are summarized in Table 4. Note again that substrate density is calculated relative to the volume of the TPS sample post-drying. Thus, this quantity represents the density of the substrate in the composite, not the initial density. Densities are compared to modeled values in Figure 10. Dashed lines denote model error bounds. Virgin density increases with substrate density as expected. However, note that an increase in substrate density is offset by reduced pore volume (and, thus, less infused resin). That is, an increase in substrate density, $\Delta \varrho_{\text {sub }}$, yields a slightly smaller increase in virgin density, $\Delta \varrho_{\text {virgin }}$. From Table 2, this increase is

$$
\Delta \varrho_{\text {virgin }}=\left(1-\frac{\varrho_{\text {resin }}}{\varrho_{\text {fiber }}}\right) \Delta \varrho_{\text {sub }}
$$

which yields $\Delta \varrho_{\text {virgin }}=0.833 \Delta \varrho_{\text {sub }}$ for C-PICA. 


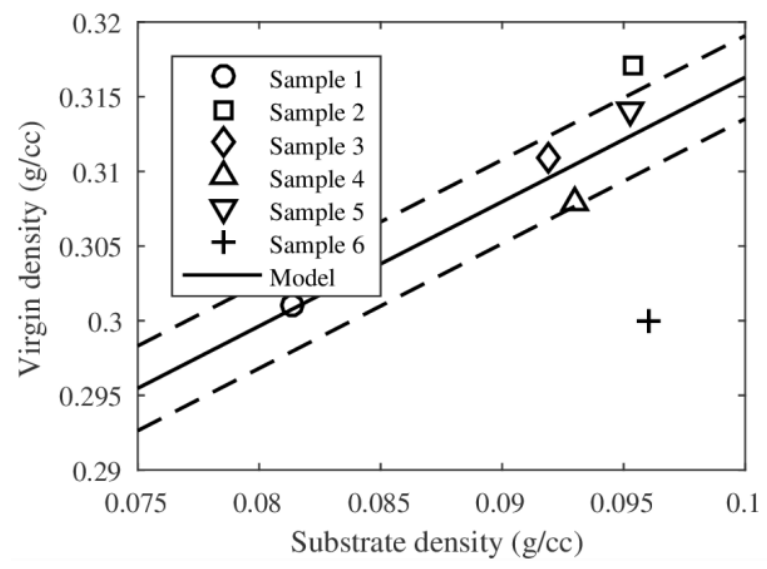

Figure 10: Virgin density as a function of substrate density.

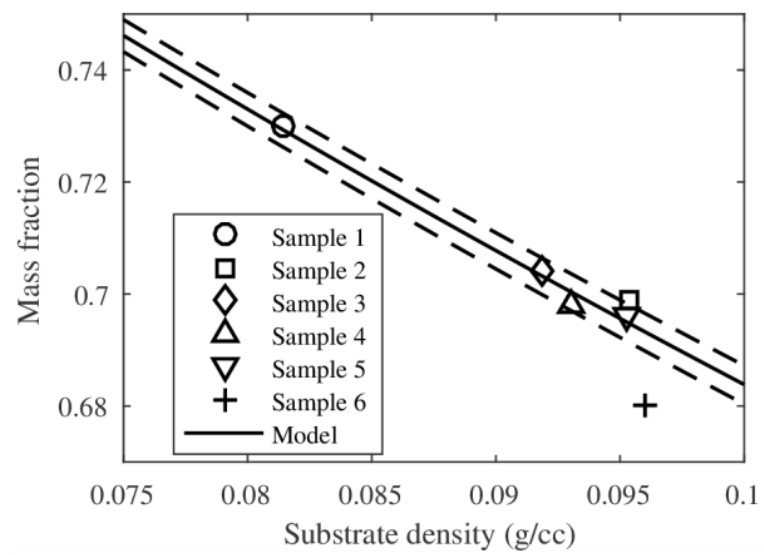

Figure 11: Resin mass fraction as a function of substrate density.

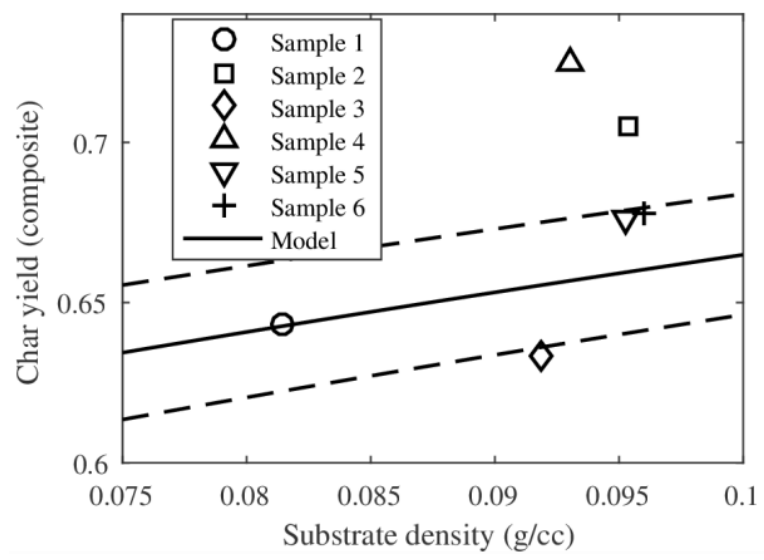

Figure 12: Composite char yield as a function of substrate density. 
The densities of Samples 3, 4 and 5 are within error bounds indicating that resin loading is comparable to Sample 1. The density of Sample 6 is substantially less than the model (and other samples) indicating that less resin is present than expected. Sample 6 clearly possesses significant voidage near the top resulting in reduced density (Figure 9, bottom right). Interestingly, Sample 5, with apparent in depth voidage (Figure 9, bottom center), possesses a nominal virgin density. Perhaps the voidage is limited to the small region pictured. Finally, the density of Sample 2 is slightly elevated indicating higher resin loading than expected, though not significantly so.

\subsubsection{Resin Mass Fraction}

Resin mass fraction follows a trend similar to virgin density (Figure 11). As before, dashed lines denote model error bounds. Mass fractions for Samples 3, 4 and 5 compare favorably to expectation. Sample 6 clearly possesses less resin than expected due to the noted voidage. Sample 2, again, shows an elevated amount of resin though not a substantial deviation from the model.

\subsubsection{Char Properties}

Post-char sample data are presented in Table 5. Samples shrink significantly more in thickness than in diameter, again due to lack of fiber connectivity through the thickness therefore TTT shrinkage is strongly influenced by resin shrinkage on charring. Resin char yields are computed assuming all mass loss is from resin decomposition. Composite char yields of the VIP-fabricated material vary from expected (Figure 12). The model assumes a nominal resin char yield, $Y_{\text {resin }}^{c}$, of $51 \pm 3 \%$ based Sample 1 results ( $\pm 3 \%$ error is currently arbitrary). All of the VIP samples, except Sample 3 , have elevated composite char yields. Samples 3, 5 and 6 possess a resin char yield that is at least comparable (within $3 \%$ ) to Sample 1. Thus, the elevated composite char yield of Sample 6 can be explained by voidage. Samples 2 and 4 do not possess any voidage, so deviation in char yield may be due to a fundamental change in the phenolic structure altering resin decomposition. Phenolic char yield is dependent on degree of crosslinking. That is, multiply bonded aromatic rings remain in the char while singly bonded rings are removed. A larger proportion of multiply bonded rings thus increases char yield. One model predicts phenolic char yield ranging from $43.2 \%$ (no crosslinks) to 65.0\% (fully crosslinked) [18]. Thus, Samples 2 and 4 may possess a greater degree of crosslinking.

Other literature, e.g. Ref Milos2015, state C-PICA resin char yield of approximately $46 \pm 1 \%$. I do not yet have an explanation of why the degree of crosslinking may have been altered here.

Table 5: Properties of cylindrical TPS coupons after charring.

\begin{tabular}{|c|c|c|c|c|c|c|c|}
\hline & \multicolumn{4}{|c|}{ Dimensions } & Mass & \multicolumn{2}{|c|}{ Char Yield } \\
\hline Sample & $D(\mathrm{~cm})$ & $t(\mathrm{~cm})$ & $\Delta D / D(\%)$ & $\Delta t / t(\%)$ & $m_{\text {total }}(\mathrm{g})$ & $Y_{\text {comp }}^{c}(\%)$ & $Y_{\text {resin }}^{c}(\%)$ \\
\hline 1 & 5.005 & 1.575 & -0.5 & -17.2 & 7.3195 & 64.3 & 51.0 \\
\hline 2 & 5.011 & 1.669 & -0.3 & -2.9 & 7.6168 & 70.5 & 57.8 \\
\hline 3 & 5.019 & 1.485 & -0.2 & -12.4 & 6.6356 & 63.3 & 48.0 \\
\hline 4 & 5.011 & 1.564 & -0.4 & -2.0 & 7.0751 & 72.5 & 60.6 \\
\hline 5 & 5.008 & 1.653 & -0.5 & -6.9 & 7.4971 & 67.6 & 53.5 \\
\hline 6 & 5.014 & 1.659 & -0.4 & -7.8 & 7.2774 & 67.8 & 52.7 \\
\hline
\end{tabular}

\section{Summary and Plan Forward}

The present work demonstrates the viability of VIP for manufacturing small C-PICA coupons. With the exception of one test (Sample 6), bulk TPS properties (virgin density and resin mass fraction) were comparable to material produced using the standard process (Sample 1). The density and resin mass fraction of Sample 2 is slightly elevated but not substantially. By visual inspection, it is clear that Samples 2 and 4 produced the highest TPS quality, with uniform resin infiltration and little or no visible voidage and delamination. Of all tests, curing conditions for these samples 
were the most similar to those of the standard process (inert at $1 \mathrm{~atm}$ ). However, the char yields for both also showed the most deviation from nominal. Additional lessons learned are summarized below. With these conclusions in mind, a plan forward for scaling the process to larger, curved tiles - of the size and shape found on an actual heat shield â€“"is presented.

\subsection{Lessons Learned}

\subsubsection{Infusion}

Infusion is a sensitive process - many aspects of the process must be carefully controlled to ensure uniform infusion and high part quality. Properties of the constituent materials (highly porous and permeable carbon felt substrate combined with a low viscosity resin solution) allow infusion to proceed easily with minimal driving pressure. However, these material characteristics lead to other unanticipated challenges. Resin advances quickly through the substrate, and solvent flashing/boiling produces bubbles in the advancing flow. While this did not appear to negatively affect uniformity in the present testing (except possibly Sample 5), the uncertainty of such an effect, especially upon scale up to larger parts, is undesirable. It is easy to imagine that voidage could be introduced as bubbles becoming trapped in the rapidly advancing flow.

Finally, the importance of proper evacuation, and outgassing, of the substrate prior to infusion cannot be understated. In the first VIP run (Sample 2), residual air may have become entrapped due to poor evacuation, later oxidizing during curing. Samples 3-6 were held under vacuum for 5-10 minutes, initially, which appeared to eliminate the problem. This outgassing period will need to be lengthened for larger parts.

\subsubsection{Curing}

Sealing the mold during curing proved to be challenging. In theory, a check valve allows volatiles generated during curing to escape without backflow of air, but this design did not work in practice. A small amount of air still leaked into the mold causing resin oxidation. Backfilling with inert gas prior to curing did not improve the result. While the oxidation did not impact the TPS part itself, there is no guarantee against such damage, so this issue must be addressed in subsequent development.

VIP processing yields nonuniformity in resin infusion at and near the TPS surface. All samples, except Sample 2, exhibited dry areas along the top surface. In two of the samples (Sample 3 and 6), solvent evaporation left small bubbles/voidage at and just below the top surface. This voidage extended farther in depth in Sample 6 than in Sample 3. Surface nonuniformity can be removed through machining. However, it is desirable to limit lost thickness because of its impact on mission design ${ }^{2}$.

Reducing ambient pressure during curing does not enhance material quality, but rather appears to degrade it. Curing at 0.66 atm appears to produce more in depth voidage and substrate delamination (Sample 5). While bulk TPS properties were not substantially altered in this particular sample, it is clear from visual inspection that material quality is substandard.

Last, closed curing seems like it may alter the char yield of the resin. Perhaps something in processing has changed the degree of crosslinking. More investigation is required. See Ref. Parker1967.

\subsection{Plan Forward}

While the results of the present testing are encouraging, VIP fabrication has yet to be demonstrated on large, curved TPS tiles. A larger scale implementation is critical to truly advancing the state of the art of conformal ablator manufacturing. Indeed, it is at large scale that VIP can meaningfully reduce process waste and labor. Mars Science Laboratory (MSL) was protected by 113 PICA tiles consisting of 27 unique designs. Tile sizes were restricted in order to limit the angle between substrate fibers and the surface tangent thus controlling variation in thermal conductivity through the thickness. Tiles were directly bonded to the composite aeroshell but required gaps and extensive gap filling due to the low strain to failure of PICA. Processes for applying gap filler had to be developed and tested. The improved properties of C-PICA allow much larger area tiles, reducing 113 PICA tiles to an estimated fewer than $40 \mathrm{C}$-PICA tiles, with zero or minimal gaps. VIP provides a means to efficiently manufacture these larger tiles. Thus, process scale up will seek to manufacture a tile representative of the size and shape possible for such a modified MSL tile design.

${ }^{2}$ Initial felt thicknesses are limited (e.g., Morgan VDG felt is available up to approximately 1 inch thick). Subsequent reductions from shrinkage and machining limit the maximum TPS thickness that can be manufactured. 
Based on lessons learned, several additions and modifications to the vacuum infusion process have been identified and will be implemented as part of scale up:

1. An outgassing step will be added prior to infusion to ensure full evacuation of the cavity and substrate.

2. Pipe and tubing diameter will be reduced to slow resin flow and reduce solvent flashing during infusion.

3. Additional thickness will be added above and below the substrate to avoid issues with solvent evaporation and surface voidage. For example, thin layers of felt can be added above and below the primary substrate and later discarded. Alternatively, a rigid, but porous, part (e.g., a mesh) could serve the same purpose but be reused.

4. Curing will be closed with a bleed of inert gas providing positive internal pressure. This modification allows curing in air while avoiding issues with air ingress in the present testing.

In addition to scale up, a code is being developed concurrently to automate conformal heatshield design. The tool will generate an optimal tile layout for a given heatshield and, using established flow simulation techniques to evaluate port placement, produce mold designs to manufacture each tile using VIP.

\section{Conclusion}

Current manufacturing for conformal ablators relies on an open liquid impregnation process adapted from PICA processing. Excess resin gets cured along with the part and discarded adding cost and labor. A closed vacuum infusion process, where resin is injected directly into the substrate, can eliminate this waste. VIP draws resin into a fibrous substrate within a rigid, matched mold by applying vacuum to the enclosure. In addition to eliminating resin waste, VIP fabrication allows introduction of process simulation and optimization, advancing the state-of-the-art of conformal TPS manufacturing design.

In this work, VIP was tested on small C-PICA samples. Carbon felt was infused with phenolic resin and then subjected to a range of curing conditions. Both closed and open curing was tested. One sample was fabricated using the standard method for comparison. Resulting TPS materials were generally well infused in depth. Voidage at and near the top surface proved to be an issue for most of the samples. However, for three of five VIP samples, this voidage did not extend far in depth. Felt delamination was a significant problem for one sample (cured under reduced pressure). Bulk physical properties (virgin density and resin mass fraction) compare favorably to the standard material. However, char yields varied from expected values. Samples 2 and 4 had elevated char yields which may result from a higher degree of crosslinking. More investigation is needed to fully understand the effect. Interestingly, these samples were the most uniformly infused from among all tests.

Lessons learned from the present testing include modifications to prevent air entrapment during infusion (an outgassing step and a change in tubing diameter) as well as an altered approach to curing. Curing will be closed but with an inert gas bleed to prevent oxidation. Additional thickness will be added above and below the primary substrate to avoid surface nonuniformity and voidage. These changes will be applied to a scaled up part - a curved tile from a heat shield geometry similar to MSL with a reduced tile count.

\section{Acknowledgements}

This work was supported through a NASA Space Technology Research Fellowship, Grant \#NNX14AL53H.

\section{Appendix}

Fiber density, $\varrho_{\text {fiber }}$, of the Morgan VDG carbon felt is estimated by a linear regression model. Following from Eq. $(1)$,

$$
1-\varphi=\left(\frac{1}{\varrho_{\text {fiber }}}\right) \varrho_{\text {sub }}=\beta \varrho_{\text {sub }}
$$

Thus, a plot of $(1-\varphi)$ vs. $\varrho_{\text {sub }}$ yields a line with slope, $\beta$, equal to the reciprocal of fiber density. Note that this fiber density ignores the central lumen (hollow space) characteristic of rayon-based carbon fibers [22]. Instead, the density computed can be interpreted as an average over the entire cross section. For the purposes of estimating pore volume according to the model presented previously, this quantity is sufficient. 


\subsection{Measurement}

Porosity and density were measured on fourteen cylindrical samples (approximately 1 " long by $0.5^{\prime \prime}$ diameter) of Morgan VDG felt. Samples spanned different locations across a single 41" by 12" piece. Eight samples were oriented in the through thickness direction; six samples were oriented in the in plane direction (Figure 13).

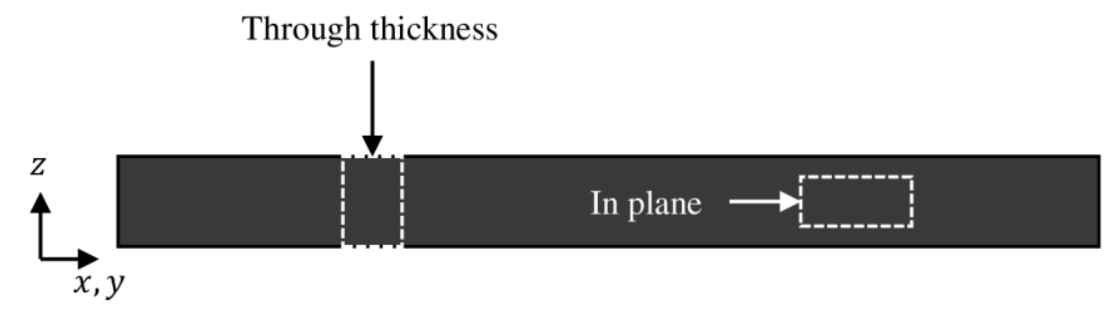

Figure 13: Illustration of through thickness and in plane sample orientations.

\subsubsection{Substrate Density}

Substrate density is computed by measuring bulk volume (sample length and width). Measurement uncertainty results in a relative error of $\pm 2 \%$ on computed densities. Additionally, the samples were found to shrink slightly in length once infiltrated, so there exists a difference between dry and saturated substrate densities. However, the difference is less than $1 \%$. This analysis uses the saturated density (consistent with the porosity measurement based on saturated samples below).

\subsubsection{Porosity}

Porosity is computed from three mass measurements: unsaturated mass of the dry sample, $m_{\mathrm{dry}}$; saturated mass of sample infiltrated with a liquid, $m_{\text {sat }}$; and suspended mass, $m_{\text {sus }}$. The latter is the measured mass increase when the saturated sample is suspended in a beaker full of the infiltrant (Figure 14). Note that the mass of the submerged sample is not being measured here, but rather the mass of the beaker. The buoyancy of the sample increases the apparent mass of the beaker (due to an equal and opposite reaction). From the first two masses, pore volume is simply,

$$
V_{\text {pore }}=\frac{m_{\text {sat }}-m_{\text {dry }}}{\varrho_{\text {liq }}}
$$

where $Q_{\text {liq }}$ is the density of the infiltrating liquid. The suspended mass is the sum of infiltrant mass and the buoyant force acting on the fibers,

$$
m_{\text {sus }}=\left(m_{\text {sat }}-m_{\text {dry }}\right)+Q_{\text {liq }} V_{\text {fiber }}
$$

which when rearranged gives an expression for the volume of the fibers

$$
V_{\text {fiber }}=\frac{m_{\text {sus }}-m_{\text {sat }}+m_{\text {dry }}}{\varrho_{\text {liq }}}
$$

Finally, the (average) porosity of the sample is 


$$
\varphi=\frac{V_{\text {pore }}}{V_{\text {pore }}+V_{\text {fiber }}}=\frac{m_{\text {sat }}-m_{\text {dry }}}{m_{\text {sus }}}
$$

Note that the dependence on infiltrant density is removed. In this work, samples were infiltrated with water. Infiltration was performed under vacuum to avoid trapping air within the sample.

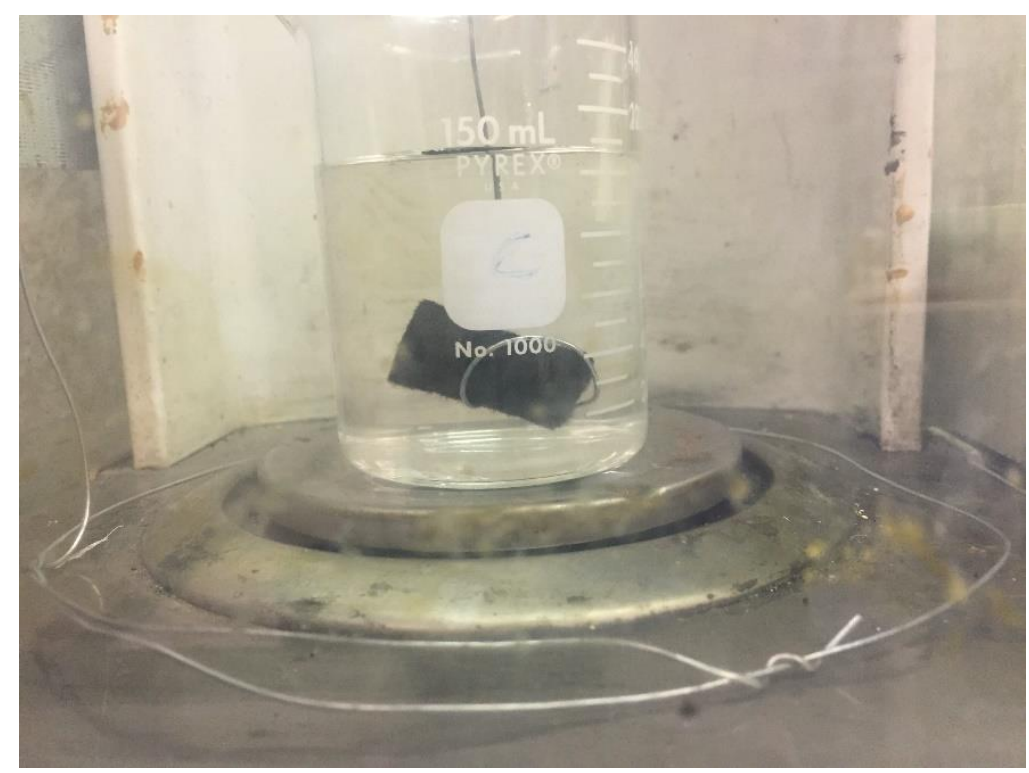

Figure 14: Saturated sample suspended in infiltrating liquid.

\subsection{Results}

Sample data and the least squares regression line are plotted in Figure 15 (left). The nominal measured values of density are shown. Bounds on the regression line depict a $95 \%$ confidence interval on the slope. The data are generally linear as predicted except for two potential outliers. Both through thickness and in plane samples appear to follow the same linear trend, though they are grouped by density. Through thickness samples possess lower densities than the in plane samples. This is likely a result of taking like samples from the same region of the felt. 


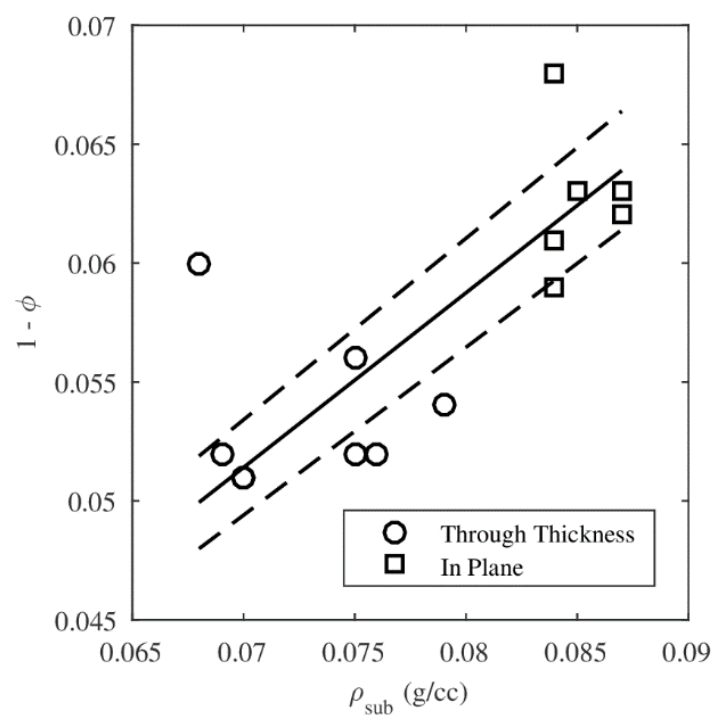

Full dataset

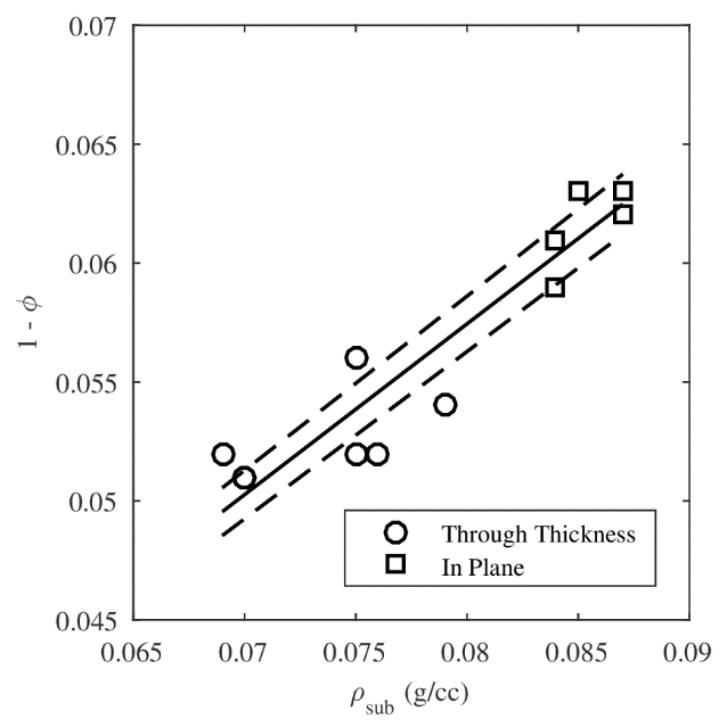

Outliers removed

Figure 15: Regression analysis on substrate samples (nominal densities).

Both outliers are biased toward higher values of $(1-\varphi)$ (thus, lower values of porosity, $\varphi$ ). This result may indicate that these samples were not fully infiltrated. Data are plotted with outliers removed in Figure 15 (right). Removing the outliers produces a slight change in slope as well as a smaller variance. As performed, this single regression assumes no error in the independent variable $\left(\varrho_{\text {sub }}\right)$. To account for error in measured densities, regression was repeated with $\varrho_{\text {sub }}$ adjusted to its maximum and minimum values (based on relative error of $\pm 2 \%$ ), which yields worst-case bounds on the slope. Results for each of these regression analyses (all with outliers removed) are summarized in Table 6. Recall that fiber density is the reciprocal of the slope. Based on maximum and minimum bounds across all analyses, fiber density lies between $1.34 \mathrm{~g} / \mathrm{cc}$ and $1.45 \mathrm{~g} / \mathrm{cc}$. This range is expressed as $\varrho_{\text {fiber }}=1.395 \pm 0.055 \mathrm{~g} / \mathrm{cc}$.

Table 6: Results of regression analyses with $95 \%$ confidence intervals.

\begin{tabular}{c|cc|cc}
\hline & \multicolumn{2}{|c|}{$\begin{array}{c}\text { Regression } \\
\text { Coefficient }\end{array}$} & \multicolumn{2}{c}{ Fiber Density } \\
Case & $\beta \quad \pm(95 \% \mathrm{CI})$ & $\mathrm{Q}_{\text {fiber }}(\mathrm{g} / \mathrm{cc})$ & $\pm(95 \% \mathrm{CI})$ \\
\hline Nominal Density & 0.718 & 0.015 & 1.39 & 0.03 \\
Maximum Density & 0.704 & 0.014 & 1.42 & 0.03 \\
Minimum Density & 0.733 & 0.015 & 1.37 & 0.03 \\
\hline
\end{tabular}

\section{References}

[1] Gasch, M., Stackpoole, M., White, S., and Boghozian, T., "Development of Advanced Conformal Ablative TPS Fabricated from Rayon- and Pan-Based Carbon Felts," 57th AIAA/ASCE/AHS/ASC Structures, Structural Dynamics, and Materials Conference, American Institute of Aeronautics and Astronautics, Reston, Virginia, jan 2016.

[2] Beck, R. A. S., Gasch, M. J., and Calomino, A., "An overview of NASA's current materials development efforts for Mars EDL," 8th International Planetary Probe Workshop, Portsmouth, VA, jun 2011.

[3] Arnold, J. O., Venkatapathy, E., and Beck, R. A. S., "Flexible Ablators: Applications and Arcjet Testing," 8th International Planetary Probe Workshop, Portsmouth, VA, jun 2011. 
[4] Beck, R. A. S., Arnold, J. O., Gasch, M. J., Stackpoole, M., Prabhu, D., Szalai, C. E., Wercinski, P., and Venkatapathy, E., "Conformal Ablative Thermal Protection System for Planetary and Human Exploration Missions: An Overview of the Technology Maturation Effort," 10th International Planetary Probe Workshop, San Jose, CA, jun 2013.

[5] Gasch, M., Agrawal, P., and Beck, R., "Testing of Advanced Conformal Ablative TPS," 10th International Planetary Probe Workshop, San Jose, CA, 2013.

[6] Milos, F. S., Gasch, M. J., and Prabhu, D. K., "Conformal Phenolic Impregnated Carbon Ablator Arcjet Testing, Ablation, and Thermal Response," Journal of Spacecraft and Rockets, Vol. 52, No. 3, 2015, pp. 804812.

[7] Venkatapathy, E., Feldman, J., Beck, R., Gage, P., Wercinski, P., Ellerby, D., and Munk, M., "Development Challenges of Game-Changing Entry System Technologies From Concept to Mission Infusion," IEEE Aerospace Sciences, Big Sky, MT, 2016.

[8] Beck, R. A. S., Arnold, J. O., Gasch, M. J., Stackpoole, M., Wercinski, P., Venkatapathy, E., Fan, W., Thornton, J., and Szalai, C. E., "Conformal Ablative Thermal Protection System for Planetary and Human Exploration Missions," 9th International Planetary Probe Workshop, No. June, Toulouse, France, jun 2012. [9] Beck, R. A. S., Arnold, J. O., Gasch, M. J., Stackpoole, M., and Venkatapathy, E., "Development of a Conformal Ablative Backshell Thermal Protection System for Outer Planetary Exploration Missions," Outer Planets Assessment Group, No. July, Bethesda, MD, jul 2014.

[10] Beck, R., Gasch, M., Stackpoole, M., Wilder, M., Boghozian, T., Prabhu, D., Kazemba, C., and Venkatapathy, E., "Conformal Ablative Thermal Protection Systems (CA-TPS) for Venus and Saturn Backshells," Outer Planets Advisory Group, 2016.

[11] Wright, M. J., Hughes, M., Calomino, A., and Barnhardt, M. D., “An Overview of Technology Investments in the NASA Entry Systems Modeling Project," 53rd AIAA Aerospace Sciences Meeting, No. January, Kissimmee, FL, 2015.

[12] Tran, H. K., Henline, W. D., Rasky, D. J., and Riccitiello, S. R., "Low-density resin impregnated ceramic article having an average density of 0.15 to $0.40 \mathrm{~g} / \mathrm{cc}$," 1996.

[13] Rudd, C. D., Long, A. C., Kendall, K. N., and Mangin, C. G. E., Liquid Moulding Technologies, Elsevier, Cambridge, England, 1997.

[14] Muskat, I., "Method of Molding," 1950.

[15] Williams, C., Summerscales, J., and Grove, S., "Resin infusion under flexible tooling (RIFT): a review," Composites Part A: Applied Science and Manufacturing, Vol. 27, No. 7, 1996, pp. 517-524.

[16] Potter, K., "The early history of the resin transfer moulding process for aerospace applications." Composites Part A: Applied Science and Manufacturing, Vol. 30, No. 5, may 1999, pp. 619-621.

[17] Parnas, R. S., Liquid Composite Molding, Hanser Gardner, Cincinnati, Ohio, 2000.

[18] Parker, J. A. and Winkler, E. L., "The effects of molecular structure on the thermochemical properties of phenolics and related polymers," Tech. rep., 1967.

[19] González-García, J., Bonete, P., Expósito, E., Montiel, V., Aldaz, A., and Torregrosa-Maciá, R., "Characterization of a carbon felt electrode: structural and physical properties," Journal of Materials Chemistry, Vol. 9, No. 2, 1999, pp. 419-426.

[20] Pierson, H. O., "Graphite Structure and Properties," Handbook of Carbon, Graphite, Diamonds and Fullerenes, Elsevier, 1993, pp. 43-69.

[21] Morgan, P., "Properties of Carbon Fibers," Carbon Fibers and Their Composites, chap. 20, CRC Press, Boca Raton, FL, 2005, pp. 791-859.

[22] Panerai, F., Ferguson, J. C., Lachaud, J., Martin, A., Gasch, M. J., and Mansour, N. N., "Microtomography based analysis of thermal conductivity, diffusivity and oxidation behavior of rigid and flexible fibrous insulators," International Journal of Heat and Mass Transfer, Vol. 108, 2017, pp. 801-811. 